\title{
Changes of renal function after retrograde intrarenal surgery using flexible ureteroscope in renal stone patients
}

\author{
Yu Liu ${ }^{1 \#}$, Zhongyu Jian ${ }^{1,2 \#}$, Yucheng Ma ${ }^{1}$, Yuntian Chen ${ }^{3}$, Xi Jin ${ }^{1}$, Liang Zhou ${ }^{1}$, Kunjie Wang ${ }^{1}$, Hong Li $^{1}$ \\ ${ }^{1}$ Department of Urology, Institute of Urology (Laboratory of Reconstructive Urology), West China Hospital, Sichuan University, Chengdu, \\ China; ${ }^{2}$ West China Biomedical Big Data Center, Sichuan University, Chengdu, China; ${ }^{3}$ Department of Radiology, West China Hospital, Sichuan \\ University, Chengdu, China \\ Contributions: (I) Conception and design: Y Liu, Z Jian, L Zhou, K Wang; (II) Administrative support: X Jin, L Zhou, K Wang, H Li; (III) Provision \\ of study materials or patients: Y Ma, Y Chen; (IV) Collection and assembly of data: Y Liu, Y Ma; (V) Data analysis and interpretation: Y Liu, Z Jian, \\ Y Ma, L Zhou; (VI) Manuscript writing: All authors; (VII) Final approval of manuscript: All authors. \\ \#These authors are co-first authors. \\ Correspondence to: Kunjie Wang; Liang Zhou. Department of Urology, Institute of Urology (Laboratory of Reconstructive Urology), West China \\ Hospital, Sichuan University, No. 37 Guo Xue Xiang, Chengdu 610041, China. Email: wangkj@scu.edu.cn; 694098320@qq.com.
}

Background: Retrograde intrarenal surgery (RIRS) is widely performed for renal stones. Theoretically, removing renal stones could prevent the deterioration of renal function. However, two studies reported that not all patients would see an increase in renal function after RIRS. The aim of our study was to evaluate the change of renal function of the operative site, and to identify predictors of improvement or deterioration of renal function after RIRS.

Methods: We retrospectively reviewed renal stones patients who received RIRS and single-photon emission computed tomography (SPECT) before and after surgery. Improved renal function was defined as the change of glomerular filtration rate (GFR) $>10 \%$ postoperatively, and that $<-10 \%$ was regarded as deteriorated renal function. Logistic and least absolute shrinkage and selection operator regression analyses were used to identify predictors for the improvement or deterioration of renal function, and predictive nomogram models were built.

Results: A total of 120 renal stone patients were included. Twenty-one (17.5\%), 79 (65.8\%) and 20 (16.7\%) patients had improved, stable and deteriorated renal function of operative site after surgery, respectively. Lower alkaline phosphatase, lower low-density lipoprotein, lower GFR of the operative site, thicker renal parenchyma, higher serum creatinine, and extracorporeal shock wave lithotripsy (SWL) history were associated with the improved renal function. The predictive accuracy of the model for the improved renal function was 0.800 . Additionally, older age, longer flexible ureteroscopic time, thinner renal parenchyma and existence of ureteral stones were risk factors for deteriorated renal function. The predictive accuracy of the model for the deteriorated renal function was 0.725 .

Conclusions: The renal function of most renal stone patients did not decrease after RIRS. For patients with potential deterioration of renal function postoperatively, urologists could shorten flexible ureteroscopic time to prevent the occurrence of this outcome.

Keywords: Renal stones; renal function; retrograde intrarenal surgery (RIRS); predictor; nomogram model

Submitted Dec 25, 2020. Accepted for publication Apr 06, 2021.

doi: $10.21037 /$ tau-20-1521

View this article at: http://dx.doi.org/10.21037/tau-20-1521 


\section{Introduction}

The incidence of renal stones increased rapidly worldwide and its prevalence was about $1-19 \%$ across the world during the past twenty years $(1,2)$. The cost of renal stones was about $\$ 3.79$ billion in 2007 in the USA, and it would increase to $\$ 4.57$ billion in 2030 (3). The recurrence of renal stones occurred in about half of patients five years after lithotripsy (2). Shock wave lithotripsy (SWL), percutaneous nephrolithotomy (PCNL) and retrograde intrarenal surgery (RIRS) are commonly used for removing renal stones. RIRS using flexible ureteroscope was recommended as the first choice for patients with renal stones $<2 \mathrm{~cm}(4,5)$. RIRS was safe that a minority of patients had complications, including postoperative fever $(8.5 \%)$, ureteral perforation $(3.5 \%)$ and urinary tract infection (2.4\%) (6).

Theoretically, removing renal stones could prevent the deterioration of renal function. However, two studies, evaluating the change of renal function after RIRS, reported that not all patients would see an increase in renal function after RIRS $(7,8)$, which may be associated with many factors, like preoperative renal function and flexible ureteroscopic time. In addition, both of them had some limitations. A study used estimated glomerular filtration rate (eGFR), which may not reflect the realistic change of renal function of operative site after RIRS (8). Based on this, another study used single-photon emission computed tomography (SPECT) to evaluating separate renal function and identified factors associated with improvement and deterioration of renal function (7). However, these predictors were inconvenient for clinical use. Besides, this study included renal stone patients receiving PCNL or RIRS, whose impacts on renal function were not the same. At present, there is limited knowledge about the effect of RIRS on renal function of operative site. If urologists could predict the change of renal function preoperatively, they could pay more attention on the related risk factors and prevent the deterioration of renal function as far as possible. The aim of this retrospective study was to evaluate the change of renal function after RIRS using SPECT in renal stone patients, identify predictors and develop nomogram model for predicting the improvement and deterioration of renal function. We present the following article in accordance with the STROBE reporting checklist (available at http://dx.doi.org/10.21037/tau-20-1521).

\section{Methods}

\section{Study design and patients}

We retrospectively reviewed renal stone patients who underwent RIRS using flexible ureteroscope in West China Hospital, Sichuan University from July 2017 to June 2019. Renal stones were diagnosed by ultrasound of the urinary system or abdominal computed tomography. All these patients had unilateral stones. Patients who received SPECT before and after surgery were included in the study. The study was conducted in accordance with the Declaration of Helsinki (as revised in 2013). The study was approved by the West China Hospital of Sichuan University Medical Research Ethics Committee approved the study (2020508) and individual consent for this retrospective analysis was waived.

\section{RIRS procedures}

All the surgeries were performed by one experienced surgeon. A 4.7 Fr double J ureteral catheter was placed two weeks before surgeries. With the patient in the lithotomic position, a 14/16 Fr ureteral access sheath was inserted into the ureter through a guidewire. Normal saline was irrigated at $160 \mathrm{cmH}_{2} \mathrm{O}$ and $0.1 \mathrm{~L} / \mathrm{min}$ by pressure pumps. Stones were fragmented using a $200 \mu \mathrm{m}$ laser fiber with holmium laser. Pulse energy and frequency for fragmenting stones were $1.5 \mathrm{~J}$ and $20 \mathrm{~Hz}$. Fragments $>2 \mathrm{~mm}$ were extracted using a nitinol basket. The remaining fragments was dusted when laser was set as $0.8 \mathrm{~J}$ and $30 \mathrm{~Hz}$. A $4.7 \mathrm{Fr}$ double J ureteral catheter was again applied at the end of the surgery, and were removed 2-3 weeks later.

\section{Data collection}

All patients received SPECT examination 12 months after surgeries. The primary outcome was the change of renal function of the operative site, which was evaluated by SPECT using ${ }^{99 \mathrm{~m}} \mathrm{Tc}-D T P A$ or ${ }^{99 \mathrm{~m}} \mathrm{Tc}-D M S A$. To compare the results of this study with that reported by Piao et al. (7), we adopted the definition of the improvement and deterioration of renal function. Improved renal function was defined as glomerular filtration rate (GFR) [operative site-contralateral site (postoperative)] - [operative sitecontralateral site (preoperative) $>10 \%$, and that $<-10 \%$ 

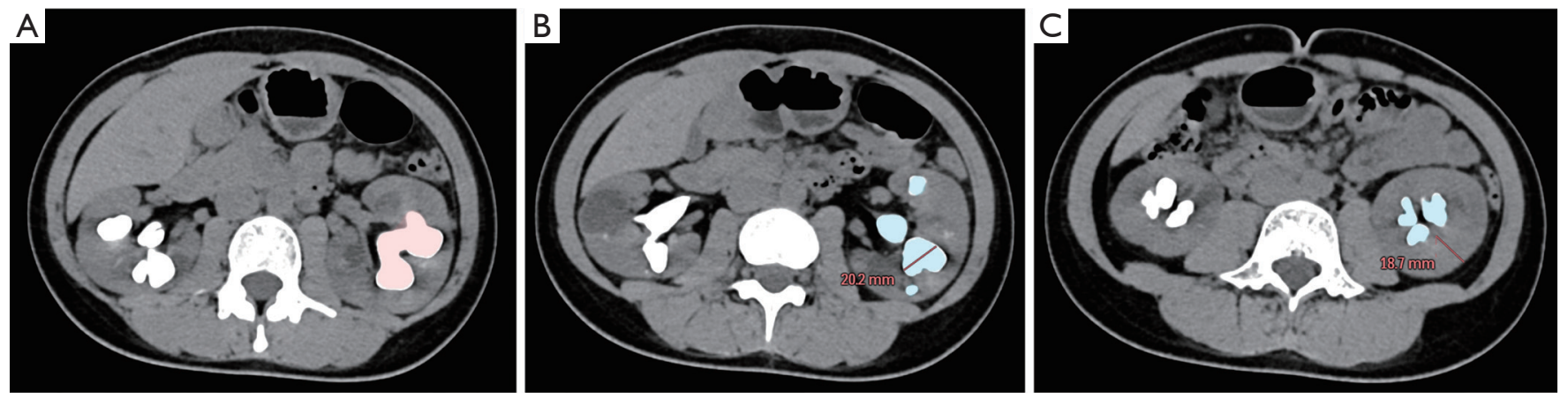

Figure 1 Segmentations of kidney on computed tomography in a renal stone patient. (A) Drawing an outline of renal stone in one segmentation and calculating its volume. (B) Calculating the maximal renal stone size of renal stone. (C) Calculating the renal parenchymal thickness.

was regarded as deteriorated renal function. The remaining patients were categorized into stable renal function group.

Preoperative factors associated with the change of renal function included demographic characteristics, disease history, operation data, laboratory data and imaging data. General characteristics were age, gender, body mass index (BMI) and history of smoking and drinking. Disease history mainly included diabetes mellitus, hypertension, hyperuricemia or gout and renal tubular acidosis. Operation data included the history of nephrolithotomy and flexible ureteroscopic time, which was defined as the time when endoscopy was inserted into the urethra. Apart from GFR, we also collected the following data, including renal parenchymal thickness, existence of hydronephrosis, ureteral stone and ureterostenosis, renal stone volume and maximal renal stone size. These data could be measures using IntelliSpace Discovery platform (Philips) (9). The schematic diagram of measuring renal stone volume, maximal renal stone size and renal parenchymal thickness were shown in Figure 1.

\section{Statistical analysis}

Mean and standard deviation were calculated for quantitative factors when they were symmetrically distributed, they were presented as mean and standard deviation, and analyzed using analysis of variance (ANOVA). Otherwise, they were shown as median and interquartile range, and compared by Kruskal-Wallis test. Categorical factors were presented as quantity and percentage, and analyzed using Chi-square test or Fisher's Exact test. We used logistic regression analysis and least absolute shrinkage and selection operator (LASSO) regression analysis to find factors associated with the improvement and deterioration of renal function, and two models for predicting the change of renal function after RIRS were also built. Nomograms of models were drawn. The sensitivity, specificity and predictive accuracy of the models were calculated. All the statistical analyses were conducted using $\mathrm{R}$ (version 3.6.3). $\mathrm{P}$ value $<0.05$ was considered as significantly different.

\section{Results}

\section{Characteristics of patients and stones}

A total of 120 patients were included in the study. About 21 (17.5\%), 79 (65.8\%) and 20 (16.7\%) patients had improved, stable and deteriorated renal function of operative site postoperatively, respectively. The mean age was $47.4 \pm$ 11.7 years, and about two-thirds of patients were males. Half of the patients had received surgeries for removing renal stones, including SWL, RIRS, PCNL and open nephrolithotomy, with no significant difference between three groups $(\mathrm{P}=0.559)$. Based on GFR of the operative site, $40.0 \%$ patients had mild renal impairment, followed by moderate renal impairment $(28.3 \%)$, normal renal function (21.7\%) and severe renal impairment (10.0\%), and GFR grade was statistically different between three groups $(\mathrm{P}=0.046)$. About half of the patients had ureteral stone or ureterostenosis. The median renal stone volume was $1.80 \mathrm{~cm}^{3}$ (Table 1). 
Table 1 Characteristics of patients and stones

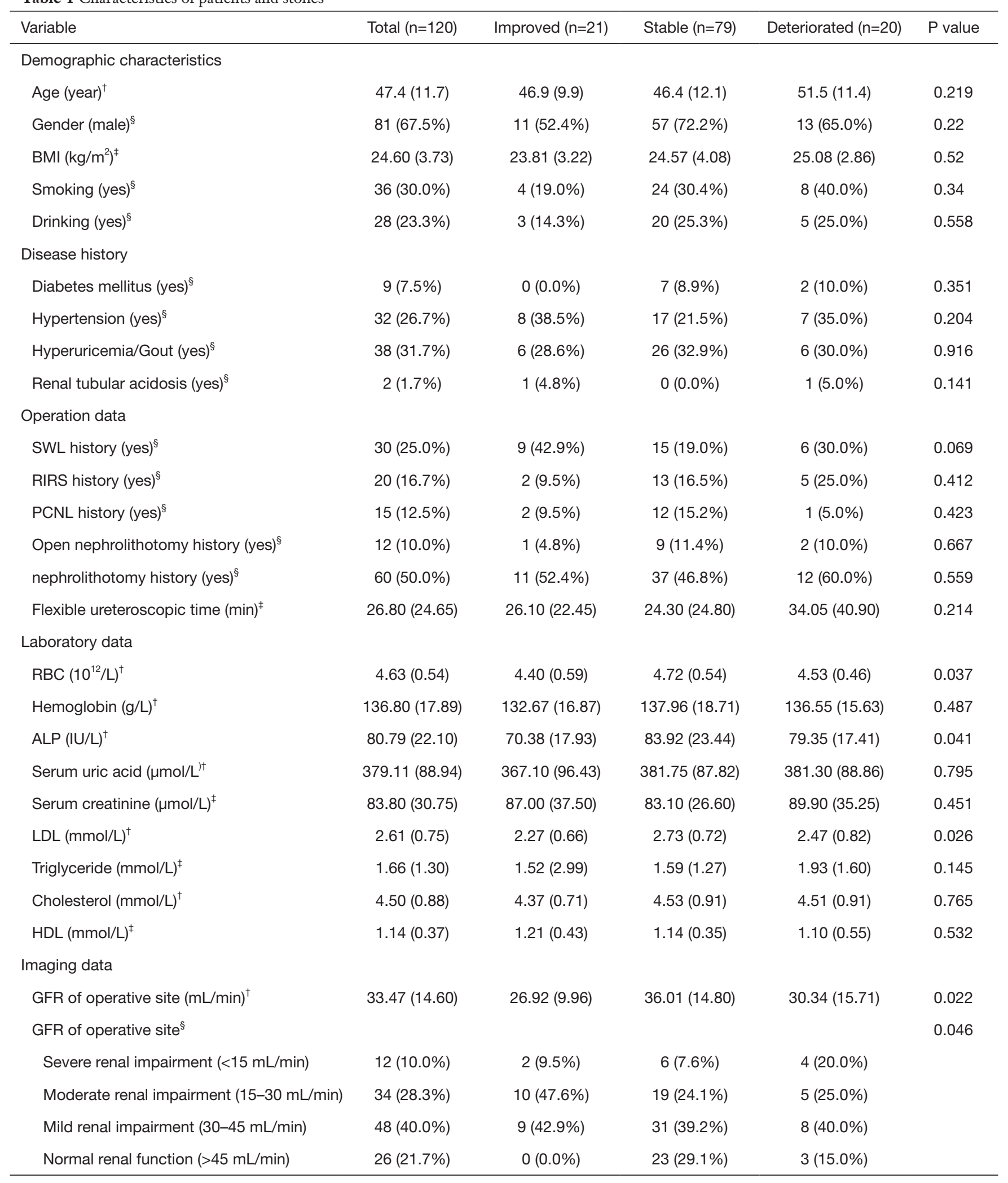

Table 1 (continued) 
Table 1 (continued)

\begin{tabular}{|c|c|c|c|c|c|}
\hline Variable & Total $(n=120)$ & Improved $(n=21)$ & Stable $(n=79)$ & Deteriorated $(n=20)$ & $P$ value \\
\hline Hydronephrosis (yes) ${ }^{\S}$ & $91(75.8 \%)$ & $18(85.7 \%)$ & $59(74.7 \%)$ & $14(70.0 \%)$ & 0.461 \\
\hline Ureteral stone $(y e s)^{\S}$ & $45(37.5 \%)$ & $8(38.1 \%)$ & $34(43.0 \%)$ & $3(15.0 \%)$ & 0.069 \\
\hline Ureterostenosis (yes) ${ }^{\S}$ & $44(36.7 \%)$ & $7(33.3 \%)$ & $30(38.0 \%)$ & $7(35.0 \%)$ & 0.913 \\
\hline Renal stone volume $\left(\mathrm{cm}^{3}\right)^{\ddagger}$ & $1.80(3.46)$ & $2.02(3.94)$ & $1.75(3.14)$ & $2.46(4.69)$ & 0.188 \\
\hline Maximal renal stone size $(\mathrm{mm})^{\ddagger}$ & $13.7(11.0)$ & $10.8(11.5)$ & $12.8(11.3)$ & $15.7(8.1)$ & 0.162 \\
\hline
\end{tabular}

${ }^{\dagger}$, data were presented as mean (standard deviation), and were analyzed with analysis of variance (ANOVA); ${ }^{\ddagger}$, data were presented as median (interquartile range), and were analyzed with Kruskal-Wallis test; ${ }^{\S}$, data were presented as quantity (percentage), and were analyzed with Chi-square test or Fisher's Exact test. BMI, body mass index; SWL, shock wave lithotripsy; RIRS, retrograde intrarenal surgery; PCNL, percutaneous nephrolithotomy; RBC, red blood cell; ALP, alkaline phosphatase; LDL, low density lipoprotein; HDL, high density lipoprotein; GFR, glomerular filtration rate.

\section{Predictors for the improved renal function after RIRS and predictive model}

We firstly performed univariate logistic regression analysis and then selected factors with $\mathrm{P}$ value $<0.100$ for multivariate logistic regression analysis. It showed that lower alkaline phosphatase (ALP), lower low-density lipoprotein (LDL), lower GFR of the operative site, thicker renal parenchymal, higher serum creatinine and SWL history were predictors for the improved renal function of the operative site after RIRS $(\mathrm{P}<0.050)$ (Table 2). Apart from these factors, LASSO regression analysis also showed that $\mathrm{BMI}$ and HDL were associated with improvement of renal function (Figure $2 A, B$ ).

Due to the limitation of sample size, we only selected six factors both occurred in logistic and LASSO regression analyses to build a model for predicting the improvement of renal function, including SWL history, ALP, GFR of the operative site, LDL, renal parenchymal thickness and serum creatinine. The area under the curve (AUC) of receiver operating characteristic (ROC) was 0.798 (Figure 2C). We also performed an internal four-fold crossvalidation for 1000 times, and the mean AUC of ROC was 0.730 (95\% CI, 0.728-0.732). To decrease misprediction rate to supervise patients' follow-up, the cut-off value of probability of improved renal function was set as 0.256 , and the sensitivity and specificity were 0.667 and 0.828 respectively, with the predictive accuracy of 0.800 . To visualize the predictive model, a nomogram was drawn (Figure 2D). Hodges-Lehmmann test showed that there was no mismatch between predictive model and retrospective cohort $\left(\chi^{2}=8.473, \mathrm{P}=0.389\right)$.

\section{Predictors for the deteriorated renal function after RIRS and predictive model}

Similarly, univariate logistic regression analysis showed that older age, larger renal stone size, longer flexible ureteroscopic time, thinner renal parenchyma and existence of ureteral stones were risk factors for deteriorated renal function after RIRS $(\mathrm{P}<0.100)$. The last three factors were still statistically different in multivariate logistic regression analysis $(\mathrm{P}<0.050)$ (Table 3$)$. In addition, LASSO regression analysis screened out factors associated with the deterioration of renal function, including age, endoscopic time and renal parenchymal thickness (Figure $3 A, B$ ).

A predictive model for deteriorated renal function was developed based on four factors both occurred in logistic and LASSO regression analyses, including age, renal parenchymal thickness, endoscopic time and ureteral stone. The AUC of ROC was 0.807 (Figure 3C). After 1,000 times cross validation, it was 0.745 (95\% CI, $0.743-0.748$ ). When the cut-off value of probability of deteriorated renal function was 0.162 , the sensitivity $(0.800)$ and specificity $(0.710)$ were high. The predictive accuracy was 0.725 . The predictive nomogram was also demonstrated (Figure $3 D$ ). Hodges-Lehmmann test indicated that indicated that the predictive accuracy of the nomogram model was good $\left(\chi^{2}=3.977, \mathrm{P}=0.859\right)$. 
Table 2 Factors associated with the improved renal function of operative site after retrograde intrarenal surgery

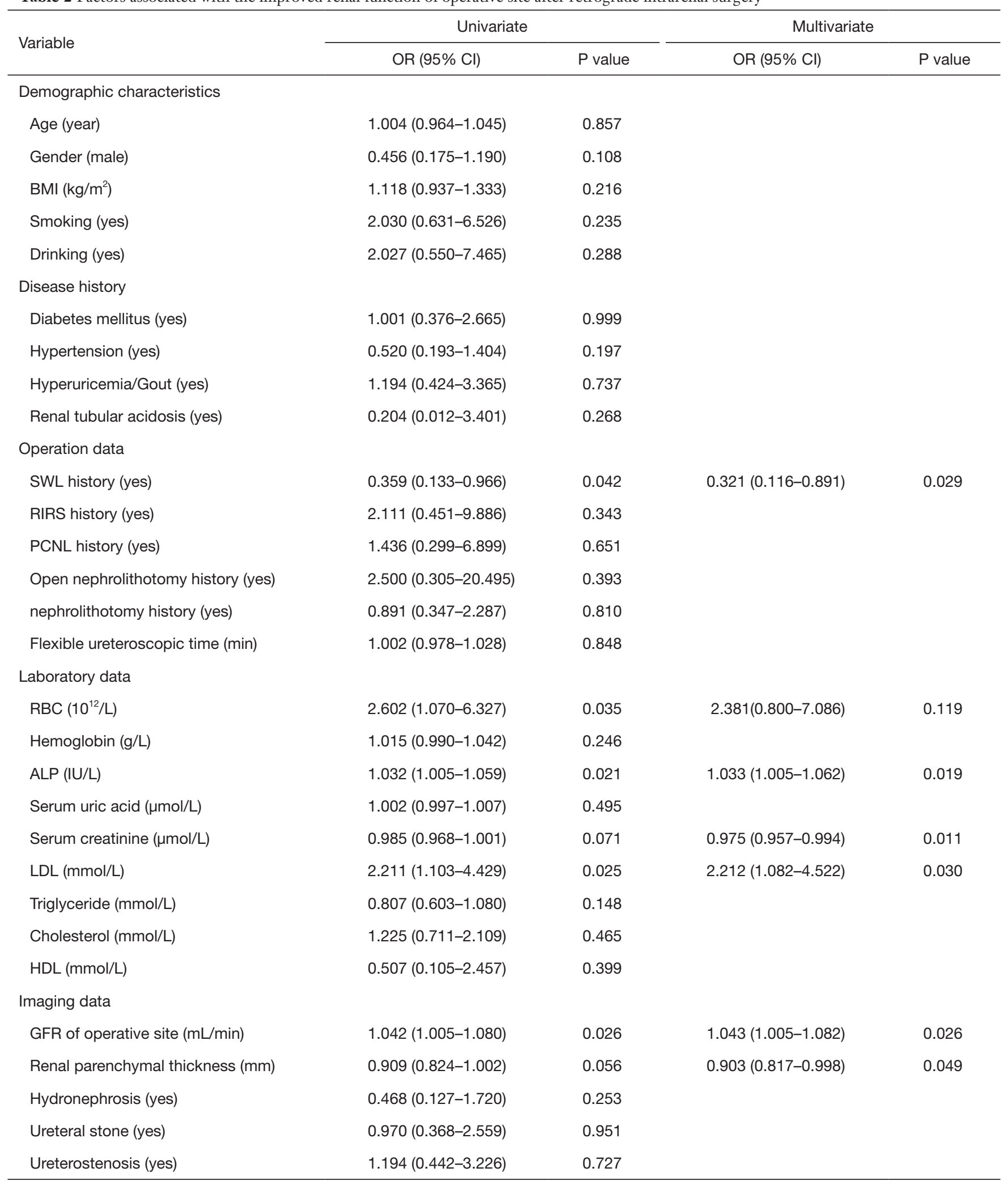

Table 2 (continued) 
Table 2 (continued)

\begin{tabular}{lccc}
\hline \multirow{2}{*}{ Variable } & Univariate & & Multivariate \\
\cline { 2 - 3 } & OR $(95 \% \mathrm{Cl})$ & $\mathrm{P}$ value & OR $(95 \% \mathrm{Cl})$ \\
\hline Ureteral stone or ureterostenosis (yes) & $1.536(0.594-3.973)$ & 0.376 \\
Renal stone volume $\left(\mathrm{cm}^{3}\right)$ & $0.989(0.902-1.084)$ & 0.814 \\
Maximal renal stone size $(\mathrm{mm})$ & $1.022(0.966-1.081)$ & 0.444 \\
\hline
\end{tabular}

BMI, body mass index; SWL, shock wave lithotripsy; RIRS, retrograde intrarenal surgery; PCNL, percutaneous nephrolithotomy; RBC, red blood cell; ALP, alkaline phosphatase; LDL, low density lipoprotein; HDL, high density lipoprotein; GFR, glomerular filtration rate; OR, odds ratio; $\mathrm{Cl}$, confidence intervals.

A

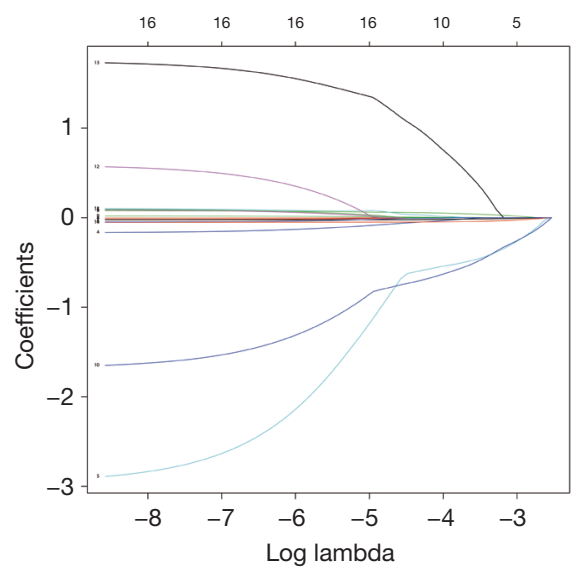

B

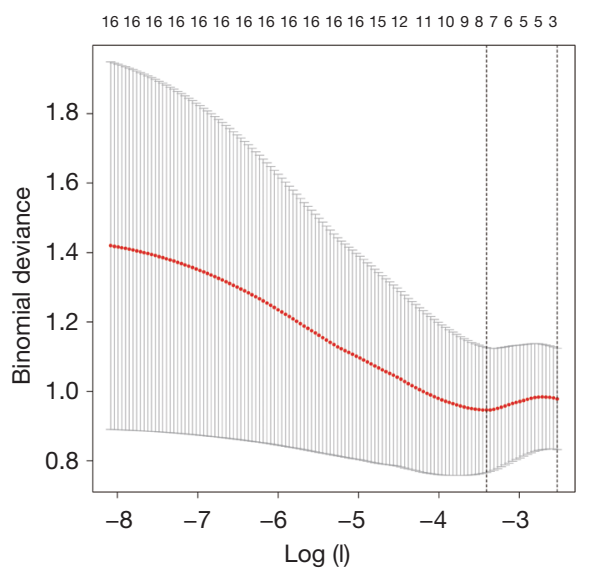

C

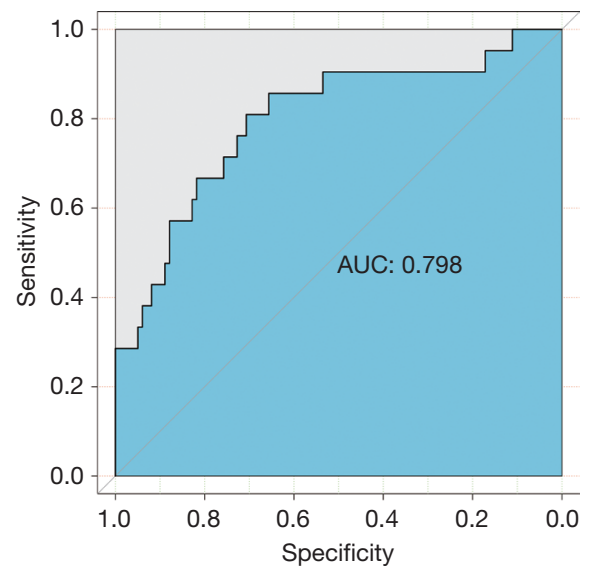

D

Points

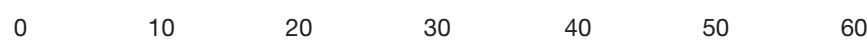

60

70

80

90

100

SWL_history

ALP

GFR_of_operative_site

LDL

Serum_creatinine

Renal_parenchymal_

thickness

Total points

Linear predictor

Probability

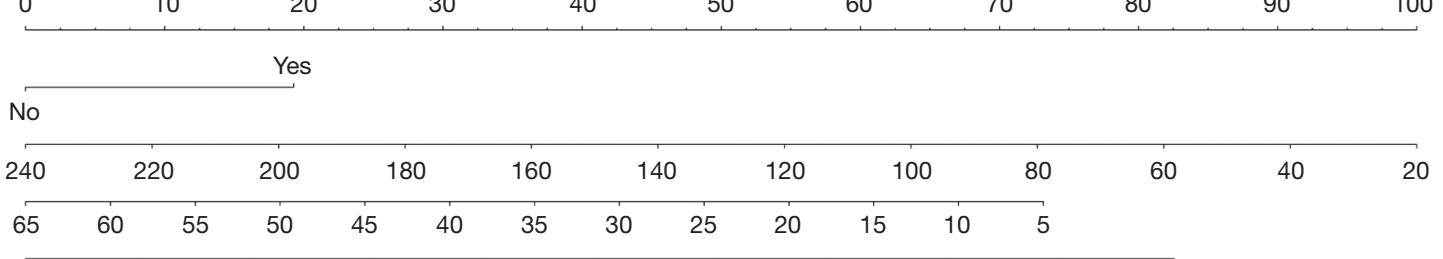

\begin{tabular}{lllllllllll}
\hline 5 & 4.5 & 4 & 3.5 & 3 & 2.5 & 2 & 1.5 & 1 & 0.5 & 0
\end{tabular}

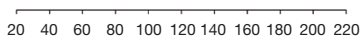

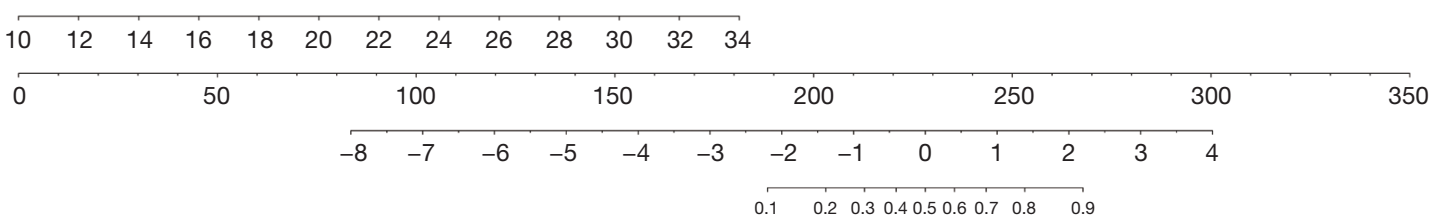

Figure 2 Predictive model for predicting the improved renal function after retrograde intrarenal surgery using flexible ureteroscope. (A) LASSO coefficient profiles of all the characteristics of patients and stones. (B) Identification of the optimal penalization coefficient Lambda in the LASSO model with 10-fold cross validation via minimum criteria. (C) Receiver operating characteristic curve of predictive model. (D) The nomogram of predictive model. LASSO, least absolute shrinkage and selection operator. 
Table 3 Factors associated with the deteriorated renal function of operative site after retrograde intrarenal surgery

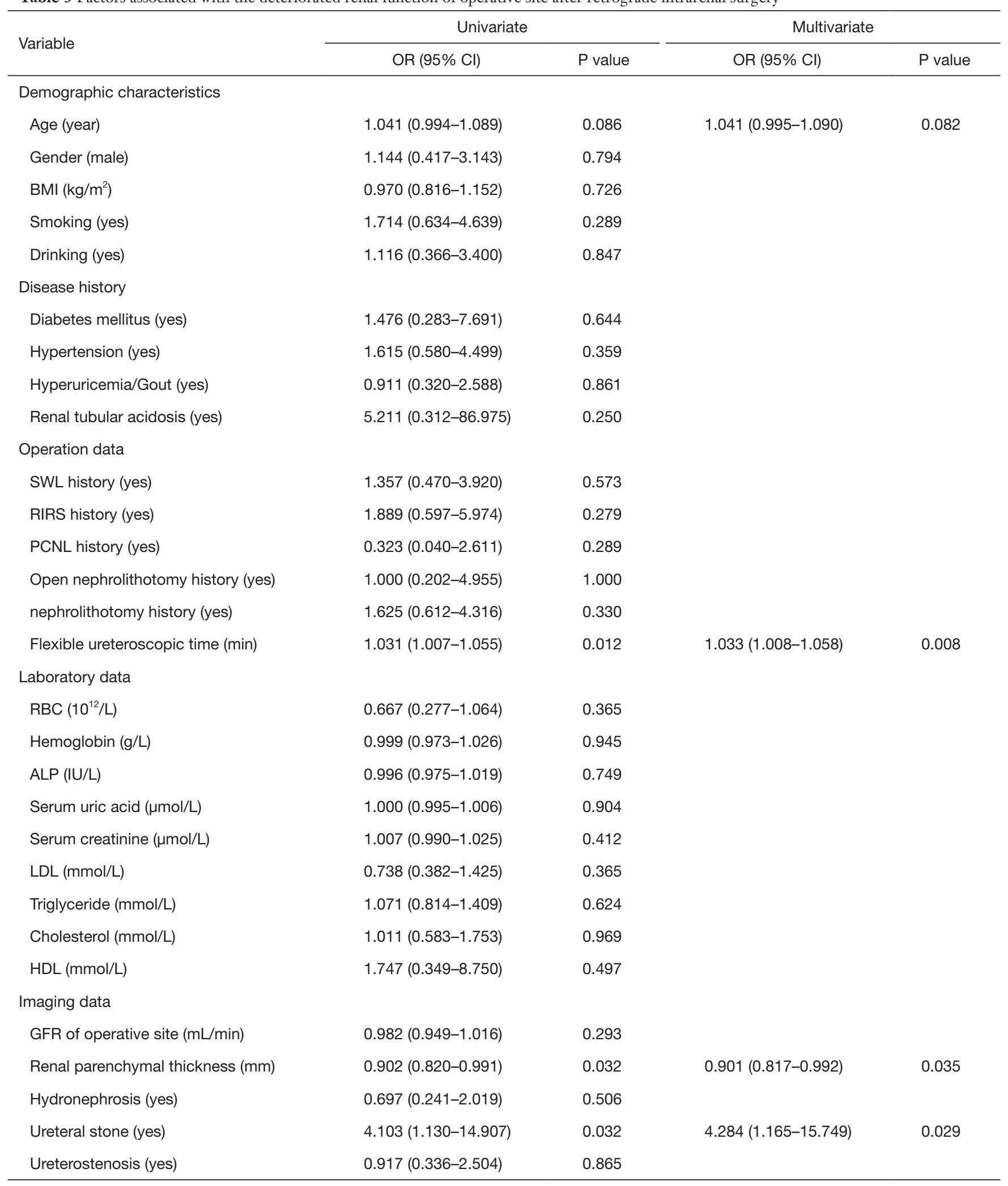

Table 3 (continued) 
Table 3 (continued)

\begin{tabular}{lccr}
\hline \multirow{2}{*}{ Variable } & Univariate & & Multivariate \\
\cline { 2 - 3 } & OR $(95 \% \mathrm{Cl})$ & $\mathrm{P}$ value & OR $(95 \% \mathrm{Cl})$ \\
\hline Ureteral stone or ureterostenosis (yes) & $0.441(0.162-1.197)$ & 0.108 & $\mathrm{P}$ value \\
Renal stone volume $\left(\mathrm{cm}^{3}\right)$ & $1.038(0.954-1.130)$ & 0.384 & \\
Maximal renal stone size $(\mathrm{mm})$ & $1.047(0.992-1.105)$ & 0.093 & $1.051(0.994-1.111)$ \\
\hline
\end{tabular}

BMI, body mass index; SWL, shock wave lithotripsy; RIRS, retrograde intrarenal surgery; PCNL, percutaneous nephrolithotomy; RBC, red blood cell; ALP, alkaline phosphatase; LDL, low density lipoprotein; HDL, high density lipoprotein; GFR, glomerular filtration rate; OR, odds ratio; $\mathrm{Cl}$, confidence intervals.

A

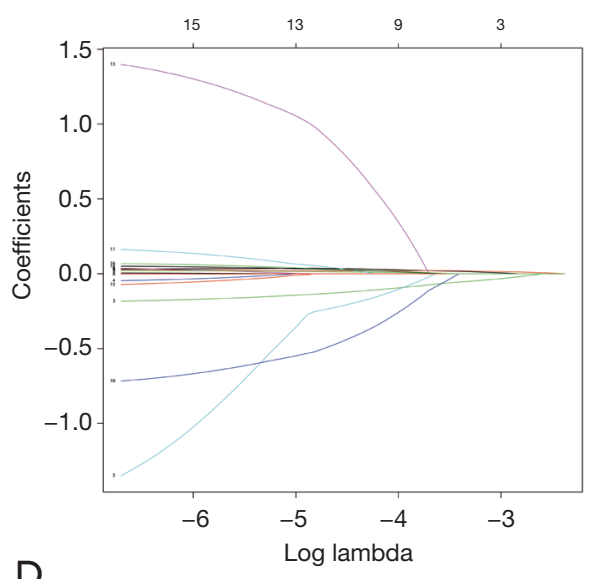

B

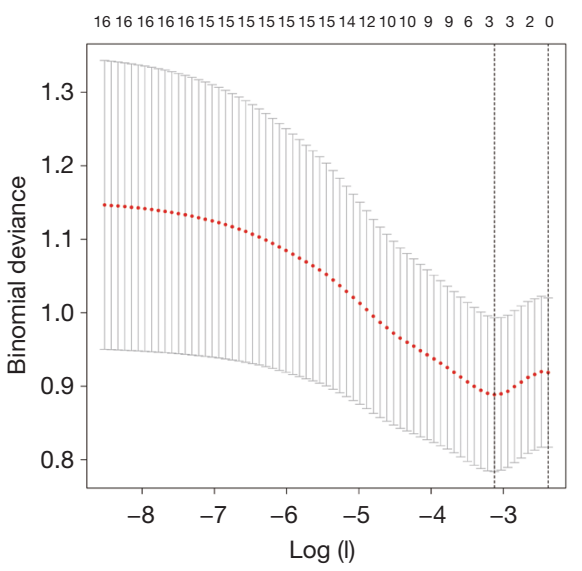

C

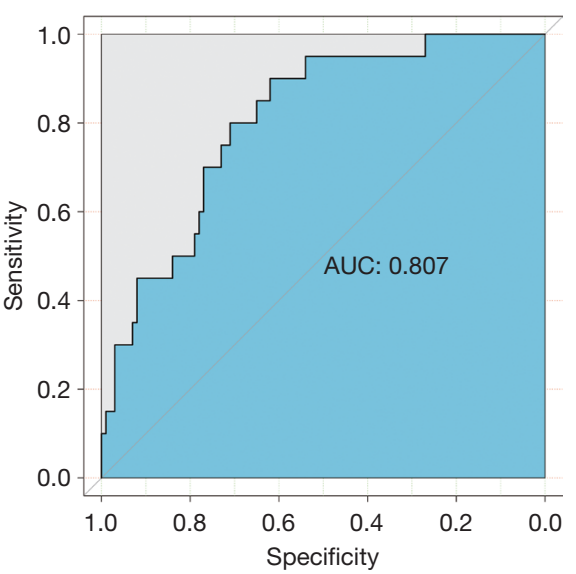

Points

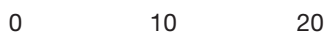

30

\begin{abstract}
40
\end{abstract}

\begin{abstract}
50
\end{abstract}

\begin{abstract}
60
\end{abstract}

\begin{abstract}
$70 \quad 80$
\end{abstract}
90

100

Renal_parenchymal_

thickness

34

28

24

22

20

18

16

10

Endoscopic_time

Ureteral_stone

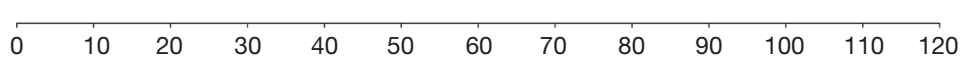

Yes

Age

Total points

Linear predictor

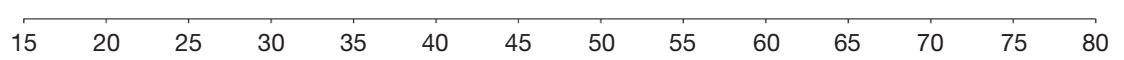

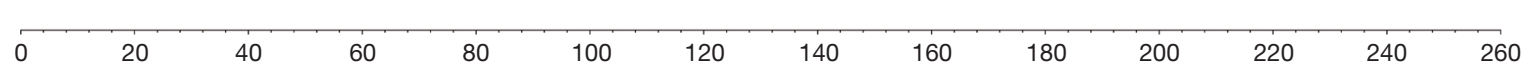

Probability

\begin{tabular}{rllllllllllllll}
\hline-5 & -4.5 & -4 & -3.5 & -3 & -2.5 & -2 & -1.5 & -1 & -0.5 & 0 & 0.5 & 1 & 1.5 & 2
\end{tabular}

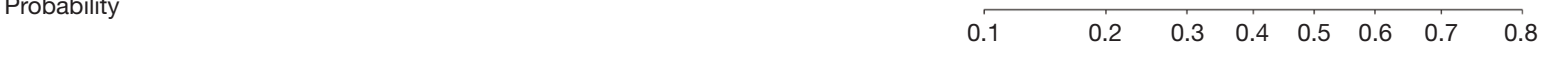

Figure 3 Predictive model for predicting the deteriorated renal function after retrograde intrarenal surgery using flexible ureteroscope. (A) LASSO coefficient profiles of all the characteristics of patients and stones. (B) Identification of the optimal penalization coefficient Lambda in the LASSO model with 10-fold cross validation via minimum criteria. (C) Receiver operating characteristic curve of predictive model. (D) The nomogram of predictive model. LASSO, least absolute shrinkage and selection operator. 


\section{Discussion}

In the study, we used SPECT to evaluated the change of renal function after RIRS and found that the improvement and deterioration of renal function of operative site occurred in $17.5 \%$ and $16.7 \%$ of patients, which were slightly different from the previous studies $(7,8)$. Piao et al. reported that the increase and decrease of renal function of operative site were seen in $58.5 \%$ and $5.7 \%$ of renal stone patients after surgery respectively (7). The examination of separate renal function and definition of its change were the same in this study and ours. The reason for higher deteriorated rate and lower improved rate in our study may be that most renal stone patients in our study had already received nephrolithotomy previously, like SWL, PCNL and RIRS. Another study found that the deterioration of renal function only occurred in $4.9 \%$ patients after surgery, which was lower than that of our study (8). This study used eGFR to evaluate renal function. When the contralateral normal kidney still had compensatory potency, eGFR may not change significantly even though renal function of operative site decreased slightly postoperatively.

SWL history was positively correlated with the improvement of renal function. Fayad et al. reported that there was no statistically significant change in GFR six months after SWL using SPECT (10). Removing renal stones using SWL delayed the deterioration of renal function in patients with nephrolithiasis (11). All these evidences verified the benefits of SWL for renal function. In addition, we found that higher ALP may prevent the improvement of renal function. ALP, an enzyme hydrolyzing pyrophosphate (a vascular calcification inhibitor), could promote heterotopic calcification (12). Thus, elevated ALP may be associated with the recurrence of renal stones, leading to further renal deterioration. We also found that LDL was low in patients with improved renal function. Jiang et al. reported that high baseline LDL was associated with a more significant decrease in eGFR (13). The patients with LDL $<2.6 \mathrm{mmol} / \mathrm{L}$ showed less progression of chronic renal disease than those with higher LDL (14). Interestingly, we found that thicker renal parenchyma promoted the improvement of renal function, while higher GFR of operative site and low serum creatinine may prevent this process. This result was not unexpectedly, because these kidneys with deteriorated function had space to improve, especially when the renal morphology was not affected. This phenomenon also occurred in the study of Piao et al. which reported that more than half of patients with abnormal separate renal function before surgery had improved renal function postoperatively (7). Nordenström et al. found that low baseline renal function was a predictor of improvement of renal function in infants and children after surgery for ureteropelvic junction obstruction (15).

When it comes to deterioration of renal function after RIRS, we found that longer flexible ureteroscopy time, existence of ureteral stones and thinner renal parenchyma may be pivotal risk factors. Lane et al. also reported that prolonged ureteroscopy time was associated with increased complication rates (16). During flexible ureteroscopy, normal saline was continuously injected into the pelvis, which increased the intrarenal pressure. Existance of ureteral stones prevented the urine from flowing smoothly, and the urine collected in the renal pelvis, followed by increasing pressure. Excessive pressure leads to renal pelvis and tubular expansion and interstitial cellular infiltration, which induced production of inflammatory cytokines and oxidative stress, followed by tubular cell apoptosis, interstitial fibrosis, nephron loss, thinner renal parenchyma and final deteriorated renal function (17-19).

Compared with previous studies, we built models with high predictive accuracy for predicting the improvement and deterioration of renal function after RIRS. Using these models, urologists could screene out patients whose renal function are more likely to decrease postoperatively and gave some suggestions on how to prevent the recurrent renal stones, like sufficient drinking water $(>2,500 \mathrm{~mL} /$ day $)$, less salt and protein intake, more fruits intake rich in citrate and more exercise. Frequent urinary ultrasonography was also important during the follow-up to supervise renal stones and hydronephrosis as it was easy to perform without any radiation. Additionally, urologists could communicate with these patients about the renal function deterioration after surgery to reduce potential medical disputes and improve physician-patient relationships.

The study had several advantages and features. First, SPECT was used to precisely measure the renal function of operative site. Second, renal stone volume was accurately measured using IntelliSpace Discovery platform (Philips), which was firstly applied for studying renal stones. Third, we developed models for predicting the change of renal function after RIRS, which may support clinical decisionmaking.

However, there were also some limitations that could not be neglected. First, the sample size was relatively small. Some patients did not routinely receive urinary 
ultrasonography postoperatively in our hospital, which resulted in the loss of follow-up of renal stone patients. Second, about a third of renal stone patients were accompanied with ureteral stones. Theoretically, ureteral stones would induce hydronephrosis, followed by the decrease of renal function. We should have evaluated the change of renal function of renal stone patients with or without ureteral stones separately. However, due to the small sample size, there may be biases when performing this subgroup analysis. More studies with large sample size are needed to tackle this problem.

\section{Conclusions}

the renal function of most renal stone patients did not decrease after RIRS. For patients with potential deterioration of renal function postoperatively, urologists could shorten flexible ureteroscopic time to prevent the occurrence of this outcome.

\section{Acknowledgments}

We thank Xiaodi Zhang [Philips (China) Investment Co., Ltd. Chengdu Branch] for the instruction of measuring renal stone volume.

Funding: This work was supported by the Project of Science and Technology Department of Sichuan Province (2018SZ0118); the Project of Sichuan Province Health Department (ZH2017-101); and 1.3.5 project for disciplines of excellence, West China Hospital, Sichuan University (ZY2016104, ZYJC18015).

\section{Footnote}

Reporting Checklist: The authors have completed the STROBE reporting checklist. Available at http://dx.doi. org/10.21037/tau-20-1521

Data Sharing Statement: Available at http://dx.doi. org/10.21037/tau-20-1521

Conflicts of Interest: All authors have completed the ICMJE uniform disclosure form (available at http://dx.doi. org/10.21037/tau-20-1521). The authors have no conflicts of interest to declare.

Ethical Statement: The authors are accountable for all aspects of the work in ensuring that questions related to the accuracy or integrity of any part of the work are appropriately investigated and resolved. The study was conducted in accordance with the Declaration of Helsinki (as revised in 2013). The study was approved by the West China Hospital of Sichuan University Medical Research Ethics Committee approved the study (2020508) and individual consent for this retrospective analysis was waived.

Open Access Statement: This is an Open Access article distributed in accordance with the Creative Commons Attribution-NonCommercial-NoDerivs 4.0 International License (CC BY-NC-ND 4.0), which permits the noncommercial replication and distribution of the article with the strict proviso that no changes or edits are made and the original work is properly cited (including links to both the formal publication through the relevant DOI and the license). See: https://creativecommons.org/licenses/by-nc-nd/4.0/.

\section{References}

1. Sorokin I, Mamoulakis C, Miyazawa K, et al. Epidemiology of stone disease across the world. World J Urol 2017;35:1301-20.

2. Liu Y, Chen Y, Liao B, et al. Epidemiology of urolithiasis in Asia. Asian J Urol 2018;5:205-14.

3. Antonelli JA, Maalouf NM, Pearle MS, et al. Use of the National Health and Nutrition Examination Survey to calculate the impact of obesity and diabetes on cost and prevalence of urolithiasis in 2030. Eur Urol 2014;66:724-9.

4. NICE Guideline - Renal and ureteric stones: assessment and management: NICE (2019) Renal and ureteric stones: assessment and management. BJU Int 2019;123:220-32.

5. Somani BK, Ploumidis A, Pappas A, et al. Pictorial review of tips and tricks for ureteroscopy and stone treatment: an essential guide for urologists from PETRA research consortium. Transl Androl Urol 2019;8:S371-80.

6. Jiang H, Yu Z, Chen L. Minimally Invasive Percutaneous Nephrolithotomy versus Retrograde Intrarenal Surgery for Upper Urinary Stones: A Systematic Review and MetaAnalysis. Biomed Res Int 2017;2017:2035851.

7. Piao S, Park J, Son H, et al. Evaluation of renal function in patients with a main renal stone larger than $1 \mathrm{~cm}$ and perioperative renal functional change in minimally invasive renal stone surgery: a prospective, observational study. World J Urol 2016;34:725-32.

8. Hoarau N, Martin F, Lebdai S, et al. Impact of retrograde flexible ureteroscopy and intracorporeal lithotripsy on kidney functional outcomes. Int Braz J Urol 2015;41:920-6. 
9. Laukamp KR, Thiele F, Shakirin G, et al. Fully automated detection and segmentation of meningiomas using deep learning on routine multiparametric MRI. Eur Radiol 2019;29:124-32.

10. Fayad A, El-Sheikh MG, Abdelmohsen M, et al. Evaluation of renal function in children undergoing extracorporeal shock wave lithotripsy. J Urol 2010;184:1111-4.

11. Yoo DE, Han SH, Oh HJ, et al. Removal of kidney stones by extracorporeal shock wave lithotripsy is associated with delayed progression of chronic kidney disease. Yonsei Med J 2012;53:708-14.

12. Zhan X, Yang Y, Chen Y, et al. Serum alkaline phosphatase levels correlate with long-term mortality solely in peritoneal dialysis patients with residual renal function. Ren Fail 2019;41:718-25.

13. Jiang S, Sun X, Gu H, et al. Age-related change in kidney function, its influencing factors, and association with asymptomatic carotid atherosclerosis in healthy individuals--a 5 -year follow-up study. Maturitas 2012;73:230-8.

14. Ozsoy RC, van der Steeg WA, Kastelein JJ, et al.

Cite this article as: Liu Y, Jian Z, Ma Y, Chen Y, Jin X, Zhou L, Wang K, Li H. Changes of renal function after retrograde intrarenal surgery using flexible ureteroscope in renal stone patients. Transl Androl Urol 2021;10(6):2320-2331. doi: 10.21037/ tau-20-1521
Dyslipidaemia as predictor of progressive renal failure and the impact of treatment with atorvastatin. Nephrol Dial Transplant 2007;22:1578-86.

15. Nordenström J, Koutozi G, Holmdahl G, et al. Changes in differential renal function after pyeloplasty in infants and children. J Pediatr Urol 2020;16:329.e1-329.e8.

16. Lane J, Whitehurst L, Hameed BMZ. Correlation of Operative Time with Outcomes of Ureteroscopy and Stone Treatment: a Systematic Review of Literature. Curr Urol Rep 2020;21:17.

17. Chevalier RL, Thornhill BA, Forbes MS, et al. Mechanisms of renal injury and progression of renal disease in congenital obstructive nephropathy. Pediatr Nephrol 2010;25:687-97.

18. Grande MT, Pérez-Barriocanal F, López-Novoa JM. Role of inflammation in túbulo-interstitial damage associated to obstructive nephropathy. J Inflamm (Lond) 2010;7:19.

19. Wu B, Brooks JD. Gene expression changes induced by unilateral ureteral obstruction in mice. $\mathrm{J}$ Urol 2012;188:1033-41. 